\title{
First report of fig cryptic virus on fig in Russia
}

\author{
Sergei Chirkov ${ }^{1,3}$ - Svetlana Tsygankova ${ }^{2}$. Sergey Rastorguev ${ }^{2} \cdot$ Irina Mitrofanova $^{1} \cdot$ Svetlana Chelombit $^{1}$. \\ Eugenia Boulygina ${ }^{2} \cdot$ Natalia Slobodova $^{2} \cdot$ Fedor Sharko $^{2}$
}

Received: 3 December 2020 / Accepted: 18 March 2021 / Published online: 22 March 2021

(c) Società Italiana di Patologia Vegetale (S.I.Pa.V.) 2021

Keywords Ficus carica L. · Ficus palmata Forrsk. · Fig cryptic virus · High-throughput sequencing

Twenty-nine mosaic-affected fig trees in the cultivar collection of the Nikita Botanical Gardens, Yalta, Russia, were surveyed for fig viruses (Preising et al. 2020) in June 2020. Three Ficus carica L. and one F. palmata Forssk. trees were tested positive for fig cryptic virus (FCV). RT-PCR with primer sets R1s/a and R2s/a (Elbeaino et al. 2011), targeting ORF1 and ORF2, generated expected PCR products of 351 and $373 \mathrm{bp}$, respectively. High-throughput sequencing (HTS) of dsRNA from FCV-infected leaves was performed on MiSec Illumina platform. Contigs were assembled de novo using metaSpades program (Nurk et al. 2017). In each of four samples, two FCV-related contigs sized from 1402 to 1685 nucleotides and five or six fig mosaic virus (FMV)-related contigs 1198 to 7039 nucleotides long were found by BLASTn. Sequences of PCR products and corresponding regions of FCV genome determined by Sanger method (MW558351 - MW558356) and HTS (MW291643 - MW291650), respectively, were identical. FCV-related contigs spanned 99.1 to $100 \%$ of corresponding genomic segments of the most closely related isolate BN13 (FR687854, FR687855) from Italy. Isolates F30, F31 and F33 from $F$. carica cultivars Temri, Kraps di Hersh, and Smena, respectively, were $100 \%$ identical to each other at the nucleotide level and shared 99.3 (RNA1) and 99.6\% (RNA2) identity with BN13. Isolate F28 from $F$. palmata was 96.6 (RNA1) and 95.1\% (RNA2) identical to isolates from $F$. carica at the nucleotide and 97.0 (RNA1) and $97.9 \%$

Sergei Chirkov

s-chirkov1@yandex.ru

1 Nikita Botanical Gardens National Scientific Center, Yalta 298648, Russia

2 National Research Center "Kurchatov Institute", Moscow 123182, Russia

3 Department of Virology, Faculty of Biology, Lomonosov Moscow State University, Moscow 119234, Russia
(RNA2) at the amino acid levels. As FCV is symptomless on $F$. carica (Elbeaino et al. 2011), symptoms of mosaic disease may be due to mixed infection with FMV. To the best of our knowledge, this is the first report of FCV in Russia expanding the information on its geographical distribution. Also, FCV was first found on $F$. palmata extending natural host range of the virus.

Sequence data that support the findings of this study have been deposited in GenBank with the accession codes MW291643-MW291650 and MW558351 - MW558356.

Funding Ministry of Science and Higher Education of the Russian Federation, grants no. 075-15-2019-1659, 075-15-2019-1670. This research has been supported by the Interdisciplinary Scientific and Educational School of Moscow University "Molecular Technologies of the Living Systems and Synthetic Biology".

\section{Declarations}

Conflict of interests The authors declare that there are no conflicts of interest.

\section{References}

Elbeaino T, Kubaa RA, Digiaro M, Minafra A, Martelli GP (2011) The complete nucleotide sequence and genome organization of Fig cryptic virus, a novel bipartite dsRNA virus infecting fig, widely distributed in the Mediterranean basin. Virus Genes 42:415-421. https://doi.org/10.1007/s11262-011-0581-0

Nurk S, Meleshko D, Korobeynikov A, Pevzner PA (2017) metaSPAdes: a new versatile metagenomic assembler. Genome Res 27:824-834. https://doi.org/10.1101/gr.213959.116

Preising S, Borges DF, de Queiroz Ambrosio MM, da Silva WL (2020) A fig deal: a global look at fig mosaic disease and its putative associates. Plant Dis. https://doi.org/10.1094/PDIS-06-20-1352-FE

Publisher's Note Springer Nature remains neutral with regard to jurisdictional claims in published maps and institutional affiliations. 